\title{
Power Systems Transient Stability Analysis via Optimal Rational Lyapunov Functions
}

\author{
Dongkun Han, Ahmed El-Guindy, and Matthias Althoff \\ Department of Informatics, Technical University of Munich, 85748 Garching, Germany \\ Email: dongkun.han@tum.de, ahmed.elguindy@tum.de, althoff@in.tum.de
}

\begin{abstract}
Transient stability analysis is a traditional yet significant topic in power systems. In order to obtain the stability domain of the post-fault equilibrium point, the Lyapunov method is proven to be effective and efficient once a Lyapunov function has been found. This paper proposes an approach to compute the largest estimate of the Region of Attraction (ROA) of an equilibrium point for power systems by using rational Lyapunov functions. This class of Lyapunov functions is more general than quadratic Lyapunov functions and polynomial Lyapunov functions, thus embracing less-conservative results. The central idea of this paper is to reconstruct the non-polynomial power systems to an uncertain differential algebraic systems via the multi-variate truncated Taylor expansion. An iteration procedure is proposed to compute the largest estimate of the ROA by exploiting the Sum of Squares (SOS) technique and the Squared Matrix Representation (SMR). A classical power system with transfer conductances is studied to demonstrate the effectiveness of the proposed approach.
\end{abstract}

Index Terms-Lyapunov methods, region of attraction, sum of squares, power system transient stability, optimal rational Lyapunov function, multi-variate truncated Taylor expansion.

\section{INTRODUCTION}

Transient stability analysis of power systems has been extensively investigated using the direct method [1]-[3]. This method is able to prevent the computationally demanding timedomain simulation for the post-fault power grid, and provides a Region of Attraction (ROA) in which the operating point converges to the post-fault equilibrium point [4].

Amongst various kinds of the direct method, the exact ROA can be obtained via the Zubov equation method and the maximal Lyapunov function method [5]. Nevertheless, the solutions of Zubov equation and the maximal Lyapunov function are generally difficult to be found. The closest Unstable Equilibrium Point (UEP) method and controlling UEP method are viable for some specific power systems, but they demand for the stability establishment of equilibrium points on the stability boundary, and they are not immune to the secondwing uprising [6].

Alternatively, thanks to the recent development of real algebraic geometry and the Sum of Squares (SOS) technique, efficient methods are proposed for estimating the ROA based on Lyapunov function methods and polynomial approximations [7]-[10]. In [9], the state space is recast into an expanded one by replacing the nonlinear terms in the system dynamic with new variables. Using this method, Anghel, etc. propose an algorithm to construct polynomial Lyapunov functions for power systems with transfer conductances [11]. In [12], by using the S-procedure and a V-s algorithm given by [10], a polynomial Lyapunov function method is proposed for power systems with single-variate Taylor polynomial (without using any remainder).

However, existing work in transient stability analysis of power systems uses quadratic Lyapunov functions or polynomial Lyapunov functions, which are rather conservative compared to rational Lyapunov functions. This paper extends the result of [13] and proposes an approach to compute the largest estimate of the ROA by using rational Lyapunov functions. The following benefits can be provided for power systems transient stability analysis: 1) This method can be easily applied to any continuous-time power systems modeled with analytic nonlinear functions; 2) a less-conservative method is provided by using the optimal rational Lyapunov function (ORLF) compared to the fixed rational Lyapunov function and variable polynomial Lyapunov functions; 3) an efficient computation is carried out by exploiting the SMR technique which opens a path for constructing a quasi-convex optimization problem instead of a non-convex one.

\section{PREliminaries}

Notations: $\mathbb{N}, \mathbb{R}$ : natural and real number sets; $\mathbb{R}^{+}$: positive real number set; $0_{n}$ : origin of $\mathbb{R}^{n} ; \mathbb{R}_{0}^{n}: \mathbb{R}^{n} \backslash\left\{0_{n}\right\} ;|\alpha|$ : sum of all the elements of an $n$-dimensional multi-index $\alpha=$ $\left(\alpha_{1}, \ldots, \alpha_{n}\right) \in \mathbb{N}^{n}$, i.e., $|\alpha|=\alpha_{1}+\cdots+\alpha_{n} ; \alpha$ !: multiindex factorial of $\alpha$, i.e., $\alpha !=\alpha_{1} ! \ldots \alpha_{n} ! ; x^{\alpha}: x_{1}^{\alpha_{1}} \ldots x_{n}^{\alpha_{n}}$, for $x \in \mathbb{R}^{n}$ and $\alpha \in \mathbb{N}^{n} ; A^{T}$ : transpose of $A ; A>0(A \geq 0)$ : symmetric positive definite (semidefinite) matrix $A ; A \otimes B$ : Kronecker product of matrices $A$ and $B ; \operatorname{ver}(\mathscr{P})$ : set of vertices of the polytope $\mathscr{P} ; \operatorname{deg}(f)$ : degree of polynomial function $f(x)$ in $x ; \nabla f$ : gradient of $f(x)$, i.e., $\nabla f=$ $\left(\frac{\partial f}{\partial x_{1}}, \ldots, \frac{\partial f}{\partial x_{n}}\right)^{T} ;(*)^{T} A B$ in a form of SMR: $B^{T} A B . \mathcal{P}$ : the set of polynomials; $\mathcal{P}^{n \times m}$ : the set of matrix polynomial with dimension $n \times m$.

\section{A. Problem Formulation}

In this paper, let us consider power systems depicted by an autonomous set of nonlinear differential equations:

$$
\dot{x}=f(x), x \in \mathcal{D}
$$

where $x(t) \in \mathbb{R}^{n}$ denotes the state vector and $f: \mathbb{R}^{n} \rightarrow \mathbb{R}^{n}$ is a nonlinear function satisfying the locally Lipschitz condition, $x(0)=x_{\text {init }} \in \mathbb{R}^{n}$ is the initial state, $\mathcal{D} \subseteq \mathbb{R}^{n}$ is the domain. 
For brevity, the dependence of functions on time $t$ and state $x(t)$ will be omitted whenever reasonable.

In this paper, we are interested in estimating the ROA of the post-fault equilibrium point. Without loss of generality, we set the origin as the equilibrium-point of interest. First, let us introduce the definition of the ROA of the origin, i.e.,

$$
\mathcal{R}=\left\{x_{\text {init }} \in \mathbb{R}^{n}: \lim _{t \rightarrow+\infty} \chi\left(t ; x_{\text {init }}\right)=0_{n}\right\},
$$

where $\chi\left(t ; x_{\text {init }}\right)$ denotes the solution of system (1) at time $t$, starting from the initial state $x_{\text {init }}$. Since rational Lyapunov functions are more general than quadratic and polynomial ones, we aim to enlarge the sublevel set of a rational Lyapunov function to inner-approximate $\mathcal{R}$. Specifically, let $V(x)$ be a rational function of system (1)

$$
V(x)=\frac{V_{\text {num }}(x)}{V_{\text {den }}(x)}
$$

where $V_{\text {num }} \in \mathcal{P}$ and $V_{\text {den }} \in \mathcal{P}$ satisfy

$$
\begin{gathered}
\forall x \in \mathcal{D}, \lim _{\|x\| \rightarrow \infty} V(x)=\infty, \\
\forall x \in \mathcal{D} /\left\{0_{n}\right\}, V_{\text {num }}(x)>0, \text { and } V_{\text {num }}\left(0_{n}\right)=0, \\
\forall x \in \mathcal{D}, V_{\text {den }}(x)>0,
\end{gathered}
$$

and $\mathcal{D}$ is defined in (1). The sublevel set of $V(x)$ is

$$
\mathcal{V}(c)=\left\{x \in \mathbb{R}^{n}: V(x) \leq c\right\}
$$

where $c \in \mathbb{R}^{+}$. The function $v(x)$ is a Lyapunov function of system (1) for the origin if

$$
\dot{V}(x)<0, \forall x \in \mathcal{D} /\left\{0_{n}\right\} .
$$

We propose the main problem: Find the ORLF $v(x)$ whose sublevel set is the largest under-estimate of the ROA, i.e., solving

$$
\begin{aligned}
& \mu=\sup _{c, v} \rho(\mathcal{V}(c)) \\
& \text { s.t. }\left\{\begin{array}{l}
(3)-(6) \text { hold } \\
\mathcal{V}(c) \subseteq \mathcal{D}
\end{array}\right.
\end{aligned}
$$

where $\rho$ is a pre-definable measure of $\mathcal{V}(c)$. An illustration example of a Single-Machine-Infinite-Bus model is provided below for easy understanding.

Example 1: The classical power system considered for illustration is given by

$$
\left\{\begin{array}{l}
\dot{\delta}=\omega \\
\dot{\omega}=\frac{1}{M}\left(P_{m}-P_{e}^{M} \sin (\delta)+D \omega\right)
\end{array}\right.
$$

where $\delta$ is the generator rotor angle and $\omega$ is the angular velocity. Set up the inertial constant $M=0.026\left[\mathrm{~s}^{2} / \mathrm{rad}\right]$, the damping coefficient $D$ to be 0.11 [s/rad], the mechanical power input $P_{m}=1.0$ per unit and the electrical power output $P_{e}^{M}=1.35$ per unit.

We transform the state space such that the equilibrium point moves from $(0.749,0)$ to the origin. By selecting a polynomial Lyapunov function $V_{1}(x)=16 x_{1}^{4}+8 x_{1}^{2}+4 x_{1}^{2} x_{2}^{2}+x_{2}^{2}$, the sublevel set $\mathcal{V}_{1}\left(c_{1}\right)$ is shown in Fig. 1 to approximate $\mathcal{R}$ with $c_{1}=1$. Our goal is to find a rational Lyapunov function $V_{2}(x)$ satisfying (3)-(6) whose level set $\mathcal{V}_{2}\left(c_{2}\right)$ is larger than $\mathcal{V}_{1}\left(c_{1}\right)$, and in the end, find the ORLF $V^{*}(x)$ whose level set $\mathcal{V}^{*}\left(c^{*}\right)$ is the largest inner-estimate of $\mathcal{R}$.

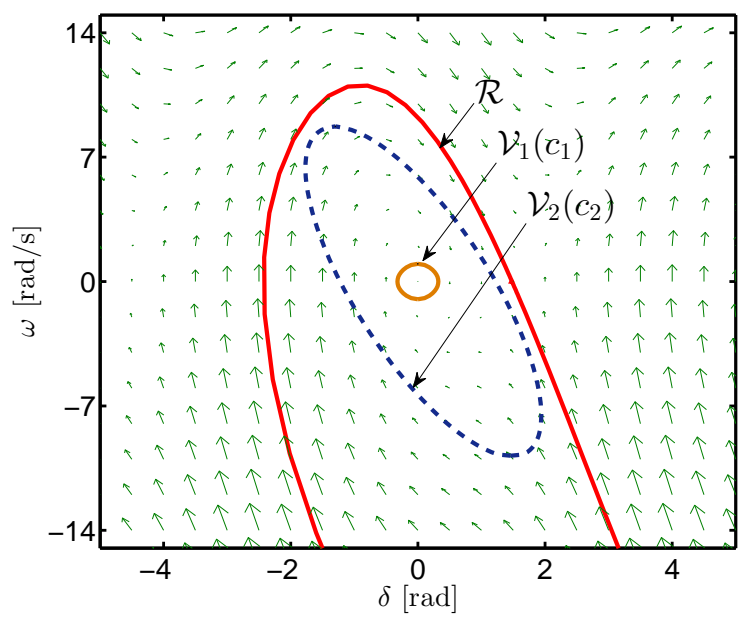

Fig. 1. Illustration example: The solid red line indicates the exact ROA of the origin; the solid yellow line and the dashed blue line indicate the boundaries of the sublevel sets $\mathcal{V}_{1}\left(c_{1}\right)$ and $\mathcal{V}_{2}\left(c_{2}\right)$, respectively.

\section{B. Basics of Sum of Squares (SOS)}

A polynomial $p(x) \in \mathcal{P}$ is nonnegative if $p(x) \geq 0$ for all $x \in \mathbb{R}^{n}$. A powerful tool for checking whether $p(x)$ is nonnegative consists of checking whether $p(x)$ can be expressed as an SOS, i.e., $p(x)=\sum_{i=1}^{k} p_{i}(x)^{2}$ for some $p_{1}, \ldots, p_{k} \in \mathcal{P}$. We denote the set of SOS polynomials as $\mathcal{P}^{\text {SOS }}$. If $p(x) \in \mathcal{P}^{\text {SOS }}$ becomes 0 only for $x=0_{n}$ and $p(x)$ is without monomials of degree 0 and 1 , we call $p(x)$ local SOS which is denoted by $\mathcal{P}_{0}^{\text {SOS }}$.

Consider a polynomial $p_{1}(x) \in \mathcal{P}_{0}^{\text {SOS }}$ of degree $\operatorname{deg}\left(p_{1}\right)$, define $d\left(p_{1}\right)$ as the smallest integer not less than $\frac{\operatorname{deg}_{x}\left(p_{1}\right)}{2}$, i.e., $d\left(p_{1}\right)=\left\lceil\frac{\operatorname{deg}_{x}\left(p_{1}\right)}{2}\right\rceil$. The SMR expression of $p_{1}(x)$ is:

$$
p_{1}(x)=(*)^{T}\left(P_{1}+L_{1}(\gamma)\right) \phi\left(n, d\left(p_{1}\right)\right)
$$

where $(*)^{T} A B$ is short for $B^{T} A B, P_{1}$ denotes the SMR matrix of $p_{1}(x), n$ is the number of variables, $\phi\left(n, d\left(p_{1}\right)\right) \in$ $\mathbb{R}^{l_{1}}$ is called the power vector containing all monomials of degree less or equal to $d\left(p_{1}\right)$ but without degree $0, L_{1}(\gamma)$ is a parameterization of the affine space

$$
\begin{aligned}
\mathscr{L}_{1}= & \left\{L_{1}(\gamma) \in \mathbb{R}^{l_{1} \times l_{1}}: L_{1}(\gamma)=L_{1}^{T}(\gamma),\right. \\
& \left.(*)^{T} L_{1}(\gamma) \phi\left(n, d\left(p_{1}\right)\right)=0\right\},
\end{aligned}
$$

in which $\gamma$ is a vector of free parameters.

\section{OPtimal RATIONAL LyAPUNOV FUNCTION APPROACH}

In this section, we first sketch the main steps of our approach. Then, each step will be explained in detail.

This approach provides a way to compute the largest estimate with a fixed Lyapunov function. Then, iteratively, a better 


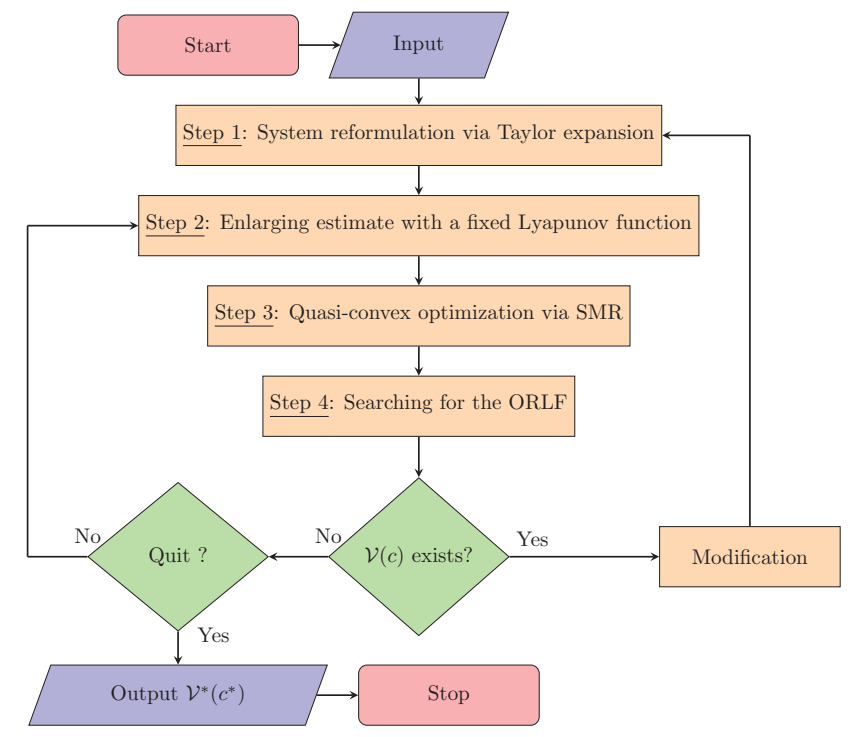

Fig. 2. Algorithm flowchart of the proposed approach.

Lyapunov function is sought and subsequently the estimate of the ROA $\mathcal{V}(\mathrm{c})$ is enlarged. The iteration procedure is shown in Fig. 2.

Step 1: System Reformulation by Using the Truncated MultiVariate Taylor Expansion

The main idea of this reformulation is to separate the polynomial functions and the non-polynomial ones, then use the truncated multi-variate Taylor expansion to approximate the non-polynomial functions. Specifically, let us equivalently rewrite the system (1) as

$$
\dot{x}(t)=h(x(t))+\sum_{i=1}^{r} g_{i}(x(t)) \zeta_{i}(x(t)), x \in \mathcal{D}
$$

where $h(x(t)), g(x(t)) \in \mathcal{P}^{n}$ are vector polynomial functions, $\zeta_{1}(x(t)), \ldots, \zeta_{r}(x(t)): \mathbb{R}^{n} \rightarrow \mathbb{R}$ denote the non-polynomial functions. We assume that $\zeta_{i}, i=1, \ldots, r$, are analytic functions within $\mathcal{D}$. Let us introduce the multi-index notations: $|\alpha|=\alpha_{1}+\cdots+\alpha_{n}, \alpha !=\alpha_{1} ! \ldots \alpha_{n} !, x^{\alpha}=x_{1}^{\alpha_{1}} \ldots x_{n}^{\alpha_{n}}$ where $x \in \mathbb{R}^{n}$ and $\alpha=\left(\alpha_{1}, \ldots, \alpha_{n}\right)^{T} \in \mathbb{N}^{n}$ is an $n$ dimensional multi-index. The $k$-th order of mixed derivatives at the origin can be expressed by $D^{\alpha} \zeta=\frac{\partial \zeta^{|\alpha|}}{\partial x^{\alpha} \ldots \partial x^{\alpha_{n}}}$, for some $|\alpha|=k$. Thus, $\zeta_{i}$ in (11) could be rewritten by the multi-variate Taylor expansion evaluated at the origin:

$$
\zeta_{i}(x)=\eta_{i}(x)+\sum_{|\beta|=k+1} \xi_{i} \frac{x^{\beta}}{\beta !}
$$

where $\xi_{i} \in \mathbb{R}$ is a bounded parameter, $k$ denotes the truncation degree and $\eta_{i}(x)$ is the $k$-th order Taylor polynomial: $\eta_{i}(x)=\left.\sum_{|\alpha| \leq k} D^{\alpha} \zeta_{i}(x)\right|_{x=0} \frac{x^{\alpha}}{\alpha !}$. We use the parameters $\xi_{i}$ to over-approximate the Taylor remainder $\zeta_{i}-\eta_{i}$, where $\xi=\left(\xi_{1}, \ldots, \xi_{r}\right)^{T}$ is in the orthotope

$$
\Xi=\left[\underline{\tau}_{1}, \bar{\tau}_{1}\right] \times \cdots \times\left[\underline{\tau}_{r}, \bar{\tau}_{r}\right]
$$

and $\underline{\tau}_{i}, \bar{\tau}_{i} \in \mathbb{R}, i=1, \ldots, r$. Please refer to [13] for the case of single-variate trauncated Taylor expansion.

Step 2: Enlarging Estimate with a Fixed Lyapunov Function

In order to solve the problem (7), one important step is to search the largest estimate of the ROA with a selected Lypaunov function, i.e., computing

$$
c^{*}=\sup c
$$

with a ratioinal function $V(x)$ such that (3)-(6) hold for all $\xi_{i} \in \Xi$, and for all $i=1, \ldots, r$. This step provides a possible solution for this problem by using the local SOS cone [13] and the real Positivstellensatz [7], [8]. In specific, the lower bound of $c^{*}$ in (14) can be obtained by handling the remainder of the Taylor expansion in a robust fashion:

$c_{k}$ is a lower bound of $c^{*}$ if there exists a polynomial $s(x) \in$ $\mathcal{P}_{0}^{\text {SOS }}$ where $c_{k}$ can be computed by

$$
\begin{aligned}
& c_{k}=\sup c_{c, s} \\
& \text { s.t. }\left\{\begin{array}{l}
-\psi(x, c, s(x), \xi) \in \mathcal{P}_{0}^{\text {SOS }} \\
\forall x \in \mathcal{V}(c) \backslash\{0\} \\
\forall \xi_{i} \in \operatorname{ver}(\Xi), i=1, \ldots, r,
\end{array}\right.
\end{aligned}
$$

in which $k$ is the truncation degree in (12),

$$
\begin{gathered}
\sigma(x)=V_{\text {den }}(x) \nabla V_{\text {num }}-V_{\text {num }}(x) \nabla V_{\text {den }} \\
r(x)=\sigma(x)\left(h(x)+\sum_{i=1}^{r} g_{i}(x) \eta_{i}(x)\right) \\
q_{i}(x)=\sigma(x) g_{i}(x) \sum_{|\beta|=k+1} \frac{x^{\beta}}{\beta !} \\
q(x)=\left(q_{1}(x), \ldots, q_{r}(x)\right)^{T} \\
\psi(x, c, s(x), \xi)=\begin{aligned}
r(x)+q(x)^{T} \xi \\
\\
+s(x)\left(c V_{\text {den }}(x)-V_{\text {num }}(x)\right),
\end{aligned}
\end{gathered}
$$

and $\operatorname{ver}(\Xi)$ is the set of vertices of $\Xi$.

Step 3: Quasi-Convex Optimization via SMR

Observe that solving (15) is not simple for the reason that there is no existing method for local SOS programming, e.g., MATLAB toolboxes YALMIP, SOSOPT and SOSTOOLS cannot handle this problem directly. To overcome these issues, the class of SMR for local SOS will be introduced, and a quasiconvex optimization problem will be formulated instead of the non-convex problem (15).

Let us introduce the SMR expressions $s(x)=$ $(*)^{T} S \phi(n, d(q)), \quad \psi(x, c, s(x), \xi)=(*)^{T}(\Psi(c, S, \xi)+$ $L(\gamma)) \cdot \phi(n, d(\psi))$, and polynomials

$$
\begin{aligned}
u(x) & =u_{1}(x)+u_{2}(x) \\
u_{1}(x) & =-r(x)-q(x)^{T} \xi+s(x) V_{\text {num }}(x) \\
u_{2}(x) & =s(x) \widetilde{V}(x) \\
\widetilde{V}(x) & =V_{\text {den }}(x)+\lambda V_{\text {num }}
\end{aligned}
$$

where $R(\xi), W(S), U_{2}(S)$ and $\widetilde{V}$ are the SMR matrix of $-r(x)-q(x)^{T} \xi, s(x) V_{\text {num }}(x), u_{2}(x)$ and $\widetilde{V}(x)$ respectively. 
For a selected truncation degree $k$, consider a positive scalar $\lambda \in \mathbb{R}^{+}$and a rational function $V(x): \mathbb{R}^{n} \rightarrow \mathbb{R}$ satisfying (3)-(4), $c_{k}$ in (15) can be computed by

$$
c_{k}=-\frac{\tilde{e}}{1+\lambda \tilde{e}}
$$

where $\tilde{e}$ is the solution of the following GEVP

$$
\begin{aligned}
& \tilde{e}=\inf _{e,} e \\
& \text { s.t. }\left\{\begin{array}{l}
S>0 \\
S U_{2}(S)>-R(\xi)-W(S)-L(\gamma) \\
\forall \xi \in \operatorname{ver}(\Xi) .
\end{array}\right.
\end{aligned}
$$

For more details of GEVP, please refer to [14].

\section{Step 4: Searching for the ORLF}

In this step, we will explain how to find the ORLF. First, let us uses the following way to obtain the initial rational Lyapunov function $V_{0}(x)$ : Select

$$
V_{0}(x)=\frac{V_{\mathrm{q}}+V_{\mathrm{a}}}{V_{\mathrm{den}}}
$$

fulfilling (4) where $V_{\mathrm{q}}(x)$ is a quadratic Lyapunov function for the linearized system of (1), and $V_{\mathrm{a}}$ is an auxiliary polynomial function which can be simply selected as $\left(x^{T} x\right) \cdot\left(x^{T} P x\right)$. We aim to find the ORLF by enlarging a selected geometric shape within $\mathcal{V}(c)$ [10]. In specific, consider

$$
\begin{aligned}
& \tilde{\mu}=\sup _{V, \epsilon} \epsilon \\
& \text { s.t. }\left\{\begin{array}{l}
\mathcal{S}(\epsilon) \subseteq \mathcal{V}(c) \\
(3)-(6) \text { hold } \\
\xi_{i} \in \Xi, \forall i=1, \ldots, r
\end{array}\right.
\end{aligned}
$$

where $\mathcal{S}(\epsilon)=\left\{x \in \mathbb{R}^{n}: \mathscr{S}(x) \leq \epsilon\right\}$ and $\mathscr{S}(x)$ is a selected polynomial, e.g., choose $\mathscr{S}(x)=\|x\|^{2}$, then the corresponding sublevel sets of $\mathcal{S}(\epsilon)$ are in a spherical shape. Similar to (15), we propose the following optimization to get a lower bound of $\rho(\mathcal{V}(c))$ :

$$
\begin{aligned}
& \bar{\mu}=\sup _{V, \epsilon, \tilde{s}} \epsilon \\
& \text { s.t. }\left\{\begin{array}{l}
\tilde{s} \in \mathcal{P}^{\mathrm{SOS}}, s \in \mathcal{P}_{0}^{\mathrm{SOS}} \\
\left(c_{k} V_{\mathrm{den}}-V_{\text {num }}\right)-\tilde{s}(\epsilon-\mathscr{S}) \in \mathcal{P}^{\mathrm{SOS}} \\
-\psi\left(x, c_{k}, s(x), \xi\right) \in \mathcal{P}_{0}^{\mathrm{SOS}} \\
\forall \xi_{i} \in \operatorname{ver}(\Xi), \forall i=1, \ldots, r .
\end{array}\right.
\end{aligned}
$$

The above problem is non-convex, and only suboptimal solution can be obtained. To solve (29), a GEVP can also be derived by using SMR technique (similar to (26)). Due to limited space, we omit here.

Note that 1) If one cannot find a $\mathcal{V}\left(c_{k}\right)$, a step of modification is needed, i.e., increase the truncation degree $k$ and the degrees of $s, \tilde{s}, V_{\text {den }}$ and $V_{\text {num. Also, set up a suitable }}$ iteration number $n_{\mathrm{it}} ; 2$ ) this approach only involves matrix inequalities consisting of GEVPs and LMIs. In addition, SMR decomposition is applied only once for the whole procedure, making this method more efficient than straightforwardly using SOS.

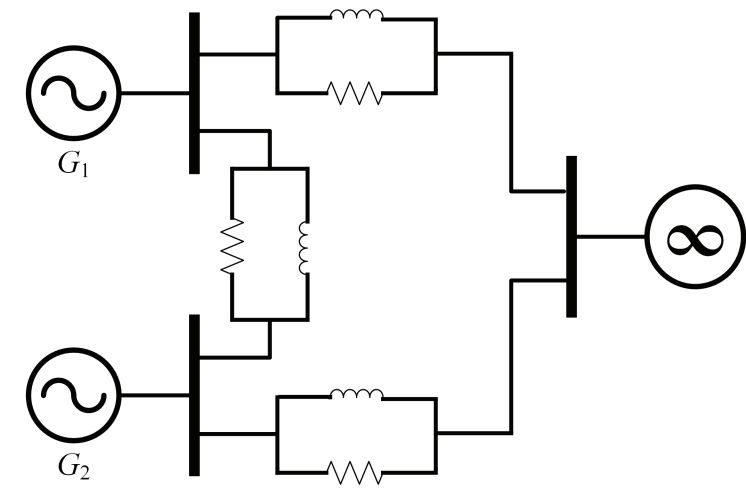

Fig. 3. Double-machine versus infinite bus power system.

\section{Case Study}

Consider a double-machine versus infinite bus power system with transfer conductances, which is shown in Fig. 3 [4], [11]. It can be expressed by

$$
\begin{aligned}
\dot{x}_{1}= & x_{2} \\
\dot{x}_{2}= & 33.5849-1.8868 \cos \left(x_{1}-x_{3}\right)-5.2830 \cos \left(x_{1}\right) \\
& -59.6226 \sin \left(x_{1}\right)-16.9811 \sin \left(x_{1}-x_{3}\right)-1.8868 x_{2} \\
\dot{x}_{3}= & x_{4} \\
\dot{x}_{4}= & 48.4810+11.3924 \sin \left(x_{1}-x_{3}\right)-3.2278 \cos \left(x_{3}\right) \\
& -99.3761 \sin \left(x_{3}\right)-1.2658 \cos \left(x_{1}-x_{3}\right)-1.2658 x_{4}
\end{aligned}
$$

where $x_{1}$ and $x_{3}$ denote the generator phase angles, $x_{2}$ and $x_{4}$ denote the angular velocities. A stable equilibrium point can be found at $(0.4680,0,0.4630,0)$. Let $y=\left(y_{1}, y_{2}, y_{3}, y_{4}\right)^{T}=$ $\left(x_{1}-0.4680, x_{2}, x_{3}-0.4630, x_{4}\right)^{T}$, we reformulate the above system in the format of system (11)

$$
\begin{aligned}
\dot{y}_{1}= & y_{2} \\
\dot{y}_{2}= & 33.5849-1.8868 y_{2}-1.8868 \eta_{1}-5.2830 \eta_{2} \\
& -59.6226 \eta_{3}-16.9811 \eta_{4} \\
\dot{y}_{3}= & y_{4} \\
\dot{y}_{4}= & 48.4810-1.2658 y_{4}-1.2658 \eta_{1}+11.3924 \eta_{4} \\
& -3.2278 \eta_{5}-99.3761 \eta_{6}
\end{aligned}
$$

where $\eta_{1}=\cos \left(y_{1}-y_{3}+0.005\right), \eta_{2}=\cos \left(y_{1}\right), \eta_{3}=\sin \left(y_{1}\right)$, $\eta_{4}=\sin \left(y_{1}-y_{3}+0.005\right), \eta_{5}=\cos \left(y_{3}\right)$, and $\eta_{6}=\sin \left(y_{3}\right)$. Let us select the initial rational Lyapunov function via (27) as

$$
V_{1}(y)=\frac{y_{1}^{2}+y_{2}^{2}+y_{3}^{2}+y_{4}^{2}+y_{1}^{4}-y_{1}^{2} y_{3}^{2}+y_{3}^{4}}{1+2 y_{1}+y_{2}-2 y_{3}+8 y_{1}^{2}+4 y_{2}^{2}+4 y_{3}^{2}},
$$

and set the truncation degree $k=5$, the shape polynomial $\mathscr{S}=y_{1}^{2}+y_{2}^{2}+y_{3}^{2}+0.5 y_{1} y_{3}+y_{4}^{2}$, the degrees $\operatorname{deg}(s)=6$ and $\operatorname{deg}(\tilde{s})=4$. We display the computation results in Tab. I with different degree combinations of rational Lyapunov functions. Let the ORLF with $\operatorname{deg}\left(V_{\text {num }}\right)=4$ and $\operatorname{deg}\left(V_{\text {den }}\right)=2$ be $V_{3}(y)$, and we compare this approach with the method of finding an optimal polynomial Lyapunov functions $V_{2}(y)$ with degree 4 [13]. The result shows a comparatively larger estimate one can obtain by using the proposed approach (see Fig. 4). 
This means that by using the ORLF approach, a better estimate of ROA can be obtained for the post-fault power system. Due to the limited space, the expressions of $V_{2}(y)$ and $V_{3}(y)$ are omitted here.

TABLE I

THE VALUES OF $c_{k}$ AND $\epsilon$ FOR SOME DEGREES OF LYAPUNOV FUNCTION AND THE CORRESPONDING COMPUTATIONAL TIME $t_{c}$.

\begin{tabular}{cccccc}
\hline $\operatorname{deg}\left(V_{\text {num }}\right)$ & $\operatorname{deg}\left(V_{\text {den }}\right)$ & $c_{k}$ & $\epsilon$ & $n_{\text {it }}$ & $t_{c}[s]$ \\
\hline 2 & 0 & 0.073 & 0.029 & 4 & 18.528 \\
2 & 2 & 1.074 & 0.673 & 6 & 53.871 \\
4 & 2 & 1.653 & 1.038 & 8 & 183.735 \\
\hline
\end{tabular}

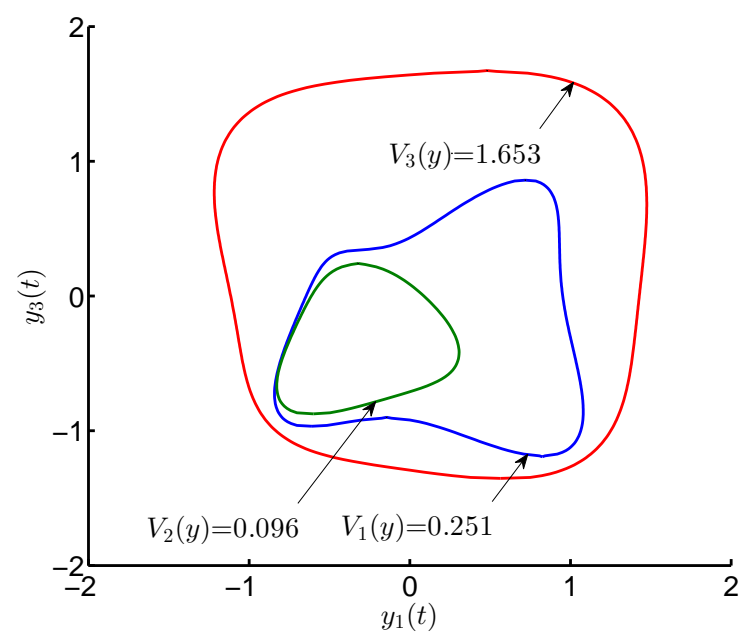

Fig. 4. The computational results shown in the angel space $y_{2}=y_{4}=$ 0.85: The solid blue line and green line indicate the specific boundaries of the sublevel sets by using $V_{1}(y)$ and $V_{2}(y)$, respectively; the solid red line indicates the boundary of the largest estimate of ROA by using the ORLF $V_{3}(y)$.

\section{CONCLUSiOn AND Future Work}

This paper provides an approach to compute the largest estimate of ROA of power systems by searching the ORLF. The multi-variate truncated Taylor expansion is exploited to reformulate the nonlinear dynamics of power system into an uncertain algebraic systems with parametric uncertainties constrained in a bounded orthotope. Then, we propose an approach to establish the estimate of the ROA by using local SOS conditions. Based on this, a quasi-convex optimization consisting of a GEVP is constructed via SMR technique. Moreover, a strategy to compute the largest estimate of the ROA is provided for searching the ORLF. Verified by a classical power system, it is shown that a larger estimate of the ROA can be obtained by the proposed method.

Like other approaches using SOS relaxation, this method also suffers from the high numerical complexity when we select high-order auxiliary functions and large-scale power system. Thus, a reasonable extension of this work is to find an optimal decomposition strategy for large-scale power systems considering the interactions between subsystems, see the pioneer work in [15]. Extra efforts would be devoted to the synthesis problem [13], the comparison with the reachability analysis [16], and the robust stability problem [17].

\section{ACKNOWLEDGMENT}

The research leading to these results is funded by the German Research Foundation (AL 1185/2-1).

\section{REFERENCES}

[1] M. Ribbens-Pavella and P. G. Murthy, Transient stability of power systems: theory and practice. John Wiley \& Sons, 1994.

[2] H.-D. Chiang, F. F. Wu, and P. P. Varaiya, "Foundations of direct methods for power system transient stability analysis," IEEE Transactions on Circuits and Systems, vol. 34, no. 2, pp. 160-173, 1987.

[3] A. Pai, Energy function analysis for power system stability. Springer Science \& Business Media, 2012.

[4] N. G. Bretas and L. F. C. Alberto, "Lyapunov function for power systems with transfer conductances: extension of the invariance principle," IEEE Transactions on Power Systems, vol. 18, no. 2, pp. 769-777, 2003.

[5] H. K. Khalil and J. W. Grizzle, Nonlinear systems. Prentice Hall Upper Saddle River, 2002.

[6] C.-C. Chu and H.-D. Chiang, "Boundary properties of the BCU method for power system transient stability assessment," in Proceedings of International Symposium on Circuits and Systems, 2010, pp. 3453-3456.

[7] P. A. Parrilo, "Structured semidefinite programs and semialgebraic geometry methods in robustness and optimization," Ph.D. dissertation, California Institute of Technology, 2000.

[8] G. Chesi, "LMI techniques for optimization over polynomials in control a survey," IEEE Transactions on Automatic Control, vol. 55, no. 11, pp. 2500-2510, 2010

[9] A. Papachristodoulou and S. Prajna, "Analysis of non-polynomial systems using the sum of squares decomposition," in Positive polynomials in control, ser. Lecture Notes in Control and Information Science. Springer, 2005, vol. 312, pp. 23-43.

[10] Z. Jarvis-Wloszek, R. Feeley, W. Tan, K. Sun, and A. Packard, "Some controls applications of sum of squares programming," in Proceedings of Conference on Decision and Control, 2003, pp. 4676-4681.

[11] M. Anghel, F. Milano, and A. Papachristodoulou, "Algorithmic construction of Lyapunov functions for power system stability analysis," IEEE Transactions on Circuits and Systems I: Regular Papers, vol. 60, no. 9, pp. 2533-2546, 2013.

[12] S. K. Mazumder and E. P. de la Fuente, "Transient stability analysis of power system using polynomial Lyapunov function based approach," in Proceedings of Power and Energy Socienty General Meeting, 2014, pp. $1-5$.

[13] D. Han and M. Althoff, "Control synthesis for non-polynomial systems: a domain of attraction perspective," in Proceedings of Conference on Decision and Control, 2015, (to appear).

[14] S. Boyd, L. El Ghaoui, E. Feron, and V. Balakrishnan, Linear matrix inequalities in system and control theory. Society for industrial and applied mathematics, 1994.

[15] M. Anghel, J. Anderson, and A. Papachristodoulou, "Stability analysis of power systems using network decomposition and local gain analysis," in Proceedings of Symposium-Bulk Power System Dynamics and Control, 2013, pp. $1-7$

[16] M. Althoff, "Formal and compositional analysis of power systems using reachable sets," IEEE Transactions on Power Systems, vol. 29, no. 5, pp. 2270-2280, 2014.

[17] D. Han and G. Chesi, "Robust synchronization via homogeneous parameter-dependent polynomial contraction matrix," IEEE Transactions on Circuits and Systems I: Regular Papers, vol. 61, no. 10, pp. 29312940, 2014. 